\title{
Influence of electric field orientation on the effect of ocular current stimulation using full field electro- retinogram
}

\begin{abstract}
Ocular current stimulation (CS) exhibits potential for the treatment of neurodegenerative ocular diseases. For a full field electroretinogram (ffERG) we found no CS effect on the characteristic waves (a-wave, b'-wave, and b-wave). To investigate whether the orientation of the generated electric field has an influence on the CS effect, this study repeated the previous ffERG study with changing one CS electrode position and compared the results of both studies. In the first study $15(8 \mathrm{~m}, 27.5 \pm 4.5$ years $)$ and in the second study $17(7 \mathrm{~m}$, $22.0 \pm 1.9$ years) healthy volunteers were stimulated with an anodal, cathodal, and sham direct CS of $800 \mu \mathrm{A}$ for $5 \mathrm{~min}$ in three sessions (randomized, different days). For both studies, a cut-sized ring rubber electrode was placed around the eye. A square rubber electrode was placed for the first study at the ipsilateral temple and for the second study at the visual cortex. Before (ERG 1) and during (ERG 2) the CS, the ffERG was measured. For both studies, the difference between the ERG 1 and ERG 2 measurement (CS effect) for the three characteristic waves was analyzed and compared between the studies. For statistical analysis, the Mann-Whitney U test with Bonferroni correction was applied $(\alpha=0.05)$. The comparison of the data distribution showed only slight differences between the studies. The Mann-Whitney $U$ test found no significant difference of the CS effect between the studies for all amplitudes and CS groups ( $p \geq 0.0055$ ). In the mean, the latency differences were smaller than the time resolution, therefore no statements for latency effects were possible. It can be concluded that the
\end{abstract}

\footnotetext{
*Corresponding author: Maren-Christina Blum: Institute of Biomedical Engineering and Informatics, Faculty of Computer Sciences and Automation, Technische Universität IImenau, Ilmenau, Germany, maren.blum@tu-ilmenau.de

Lilly Leydolph: Institute of Biomedical Engineering and Informatics, Faculty of Computer Sciences and Automation, Technische Universität IImenau, IImenau, Germany, lilly.leydolph@tu-ilmenau.de

Sascha Klee: Department of General Health Studies, Division Biostatistics and Data Science, Karl Landsteiner University of Health Sciences, Krems an der Donau, Austria, sascha.klee@kl.ac.at; Institute of Biomedical Engineering and Informatics, Faculty of Computer Sciences and Automation, Technische Universität IImenau, IImenau, Germany
}

retinal cells generating the ffERG are not affected by ocular CS in either electrode montages tested.

Keywords: full field ERG, transorbital electrical stimulation, direct current stimulation, non-invasive brain stimulation

https://doi.org/10.1515/cdbme-2021-2043

\section{Introduction}

Transcranial current stimulation (CS) is an established method for research on the human nervous system and for the treatment of neuronal disorders [1]. In recent years, stimulation of the visual system and the retina itself has gained attention as a new therapeutic modality for neurodegenerative eye diseases, e.g., glaucoma [2]. Numerous animal studies have shown positive effects of an ocular CS on function and stability of retinal nerve cells. Human studies have mainly focused on the effect of an ocular CS on different neurodegenerative eye diseases with widely varying results [3].

Until today, it is unknown which retinal cells in detail can be influenced by an ocular CS. Blum et al. 2020 [4] attempted to address this research question by examining the effects of an ocular CS on the pattern-reversal electroretinogram (PERG). There, a decreasing CS effect was found on the P50 amplitude during an anodal and cathodal ocular CS. For sham stimulation no effects were found. The PERG is a potential mainly evoked by the retinal ganglion cells, but the P50 amplitude is also slightly influenceable by preganglion cell activity. Therefore, it was not certain whether the CS effect was due to the influence of ganglion cells or preganglion cells.

This research question was investigated by a full field electroretinogram (ffERG) study in combination with an ocular CS. With a ffERG, preganglion cell activity, mainly from photoreceptors and bipolar cells, can be investigated. Here, no CS effects on the ffERG were found [5]. In this ffERG study CS was applied between the eye and the ipsilateral temple as in the PERG study. It is conceivable that the electric field orientation was better suited for influencing the retinal ganglion cells than the preganglion cells. Therefore, a 
second ffERG study according to the preceding study was made with a shifted CS electrode from the ipsilateral temple to the $\mathrm{Oz}$ position (according to the international 10-20 system for electroencephalogram electrode positions). Here again, no CS effects on the ffERG were found (unpublished data).

Even if there was no effect within a study, there could be a difference between the studies that shows an influence of the orientation of the electric field. Therefore, the aim of the presented study was to compare the two ffERG studies to investigate the influence of the orientation of the electric field on the ocular CS effect for the ffERG.
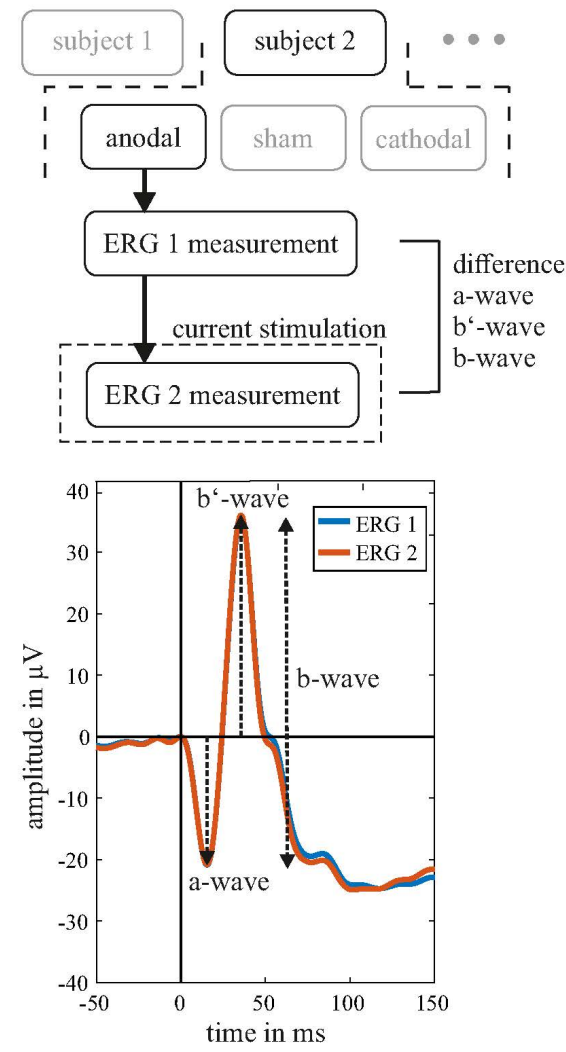

Figure 1: Measurement procedure. In both studies every volunteer went through three sessions (randomized order, different days) which differed in the type of the ocular current stimulation (CS; i.e., anodal polarity, cathodal polarity, or sham stimulation). In each session two electroretinogram (ERG) measurements were performed, one before (ERG 1) and one during (ERG 2) the CS. The difference of the characteristic waves (a-wave, b'-wave, and b-wave) between the ERG 1 and ERG 2 measurement was calculated.

\section{Material and Methods}

\subsection{Study designs}

The ffERG studies were unrelated and included different subjects. In the first ffERG study 15 healthy subjects $(8$ males, mean age: $27.5 \pm 4.5$ years) and in the second ffERG study 17 healthy subjects ( 7 males, mean age: $22.0 \pm 1.9$ years) participated.

Both studies used a monocularly presented white flash full field stimulus (flash strength: $3 \mathrm{cds} / \mathrm{m}^{2}$, duration: $\leq 5 \mathrm{~ms}$, inter stimulus interval: $0.5 \mathrm{~s}$, background illumination: none) for visual stimulation. In total, 200 artifact free sweeps were used for averaging. The characteristic waves were the awave (first negative amplitude measured from baseline), the b'-wave (first positive amplitude measured from baseline), and b-wave (measured peak-to-peak) shown in Figure 1. The ffERG was recorded using an amplifier system (Cubias-M, neuroCare Group $\mathrm{GmbH}$, Munich, Germany) with an input impedance of $\geq 10 \mathrm{G} \Omega$, a dynamic range of $\pm 170 \mathrm{mV}$, a 24 bit analog-digital converter, and a sampling rate of 2,000 samples-per-second. Sintered $\mathrm{Ag} / \mathrm{AgCl}$ ring electrodes (EASYCAP GmbH, Herrsching, Germany) were placed at the lower eyelid (active), the ipsilateral earlope (reference) and the forehead (ground) to detect the ffERG.

Direct current was applied using a battery-powered current stimulator (DC-Stimulator MC version, neuroCare Group $\mathrm{GmbH}$, Munich, Germany). In both studies a ring rubber electrode (outer/inner diameter: $75 \mathrm{~mm} / 30 \mathrm{~mm}$; thickness: $2 \mathrm{~mm}$ ) surrounded the eye and applied the current. The electrode had a cutout in the area of the lower eyelid to allow the placement of the ffERG recording electrode. The counter CS electrode was a rubber patch $\left(25 \mathrm{~cm}^{2}\right.$, thickness: $\left.2 \mathrm{~mm}\right)$ inserted into a saline-soaked $(10 \mathrm{ml})$ sponge. In the first study this electrode was positioned at the ipsilateral tempus, while in the second study it was positioned at the Oz position (Figure 2). The CS parameters were similar in both studies (current strength: $800 \mu \mathrm{A}$, duration: $5 \mathrm{~min}$ ). There were three current applications: anodal polarity, cathodal polarity, and sham stimulation. The applied current polarity (cathodal or anodal) refers to the polarity of the stimulation electrode surrounding the eye. During the sham stimulation, no current flow (current source not activated) was generated at the electrodes, but the volunteers were informed that current stimulation was performed.

In total, every volunteer in both studies underwent three sessions conducted on different days with at least one day between sessions. The three sessions only differed in the current application (i.e., anodal polarity, cathodal polarity, or sham stimulation; randomized order). In each of the three sessions two single ffERG recordings were performed: one before (ERG 1) and one during (ERG 2) the current stimulation (Figure 1). Subsequently, the difference of the ERG 1 to ERG 2 measurement for the characteristic waves of the ffERG were calculated. These difference values were compared between 
the studies for the characteristic waves and current applications.
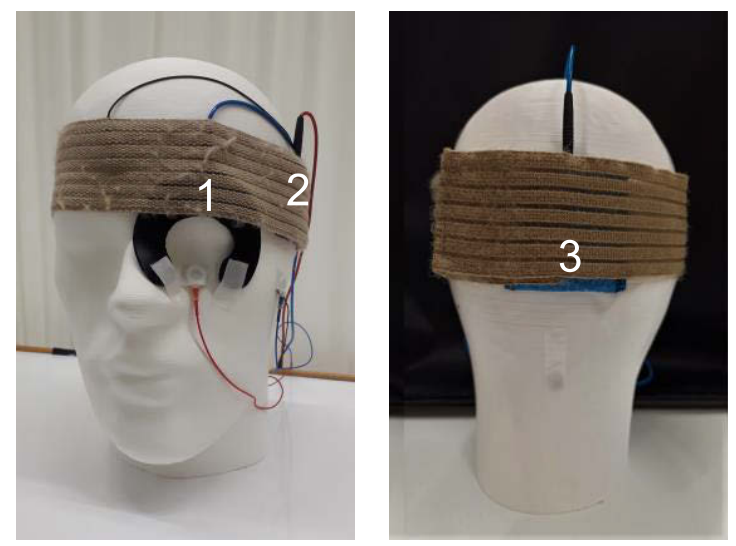

Figure 2: Electrode montage. In both studies a current stimulation (CS) electrode surrounded the eye (1). The applied current polarity (cathodal or anodal) refers to the polarity of this electrode. In the first study the counter CS electrode was positioned at the ipsilateral temple (2). In the second study the electrode position was shifted to the Oz position (3) according to the international $10-20$ system for electroencephalogram electrode positions.

\subsection{Statistical Analysis}

Statistical analysis was performed using IBM SPSS Statistics (version 25, IBM Corp., Armonk, United States). The primary aim of the presented study was to compare the differences of the ERG 1 to ERG 2 measurement between the studies for the different current applications and characteristic ffERG waves. The normal distribution hypothesis was rejected by the Shapiro-Wilk test and Q-Q plots. Therefore, the Mann-Whitney $U$ test for unrelated samples was performed between the two studies for every current application and characteristic ffERG wave. The significance level was set to $\alpha=0.05$. Based on the multiple comparison problem, the Bonferroni correction resulted in an adjusted significance value of $\mathrm{p}^{*} \leq 0.0055$.

Violin plots of the difference between the ERG 1 and ERG 2 measurement for the different current applications, ffERG waves and studies were plotted to allow a graphical analysis and evaluation of the data distribution.

\section{Results}

The data distribution (Figure 3) for the amplitude differences between the ERG 1 and ERG 2 measurement showed only slight differences between the studies. Only for the b'-wave amplitude in the cathodal as well as the $b^{\prime}$-wave and b-wave amplitude in the sham group, slightly different trends between both studies could be observed.

On average, the latency differences in both studies were smaller than the time resolution. Therefore, a latency effect analysis was not performed.

The Mann-Whitney U test revealed no significant effect between the studies (Table 1) for the comparison of the difference between the ERG 1 to the ERG 2 measurements for all current applications and all characteristic waves.

Table 1: P-values of the Mann-Whitney $U$ test. The differences of ERG 1 to ERG 2 measurement between the two studies for the different characteristic waves and current applications were compared. The significance level was set to $\alpha=0.05$ (after Bonferroni correction $\left.p^{*} \leq 0.0055\right)$

\begin{tabular}{cccc}
\hline ffERG wave & anodal & cathodal & sham \\
\hline a-wave & 0.455 & 0.027 & 0.331 \\
b'-wave & 0.823 & 0.040 & 0.069 \\
b-wave & 0.852 & 0.852 & 0.216 \\
\hline
\end{tabular}

\section{Discussion}

In the present study, two studies on the effect of ocular CS on the ffERG with different CS electrode montages were compared. In both studies the difference between the ffERG measured before (ERG 1) and during (ERG 2) direct CS of the eye for three current applications (i.e., anodal polarity, cathodal polarity, or sham stimulation) was calculated and compared between the studies. No significant difference between the studies for all characteristic ffERG waves as well as for all current applications were found.

Actual simulations to compare the current density between the used CS montages have not yet been published. However, Hunold et al. 2015 demonstrated that montages (comparing Fp2 - infraorbital to $\mathrm{Fp} 2-\mathrm{Cz}$ ) with a more distant counter electrode $(\mathrm{Fp} 2-\mathrm{Cz})$ lead to a more homogeneous current density amplitude distribution [6]. Based on this knowledge it was expected that a more homogeneous current density would reach the retina by stimulation between the eye and the $\mathrm{Oz}$ position. How much the used montages differ should be investigated in a future simulation study.

In our study it was shown that the ocular CS effect on the ffERG is independent whether oCS is performed between the eye and ipsilateral temple or between the eye and visual cortex. Therefore, it can be concluded that the effect found within the PERG study cannot be explained by an influence of the preganglion cells of the ffERG. 
The study was limited by the CS parameters and the study design. In both studies current strength and duration were set to $800 \mu \mathrm{A}$ and $5 \mathrm{~min}$ to maintain a low current load. Other current intensities and stimulation durations can have different effects [7]. In both studies the eye was not stimulated consistently because of the cutout in the ring electrode to enable the placement of one ffERG electrode at the lower eyelid. A more homogeneous current stimulation of the eye could generate other results. Furthermore, no after-effects were investigated.
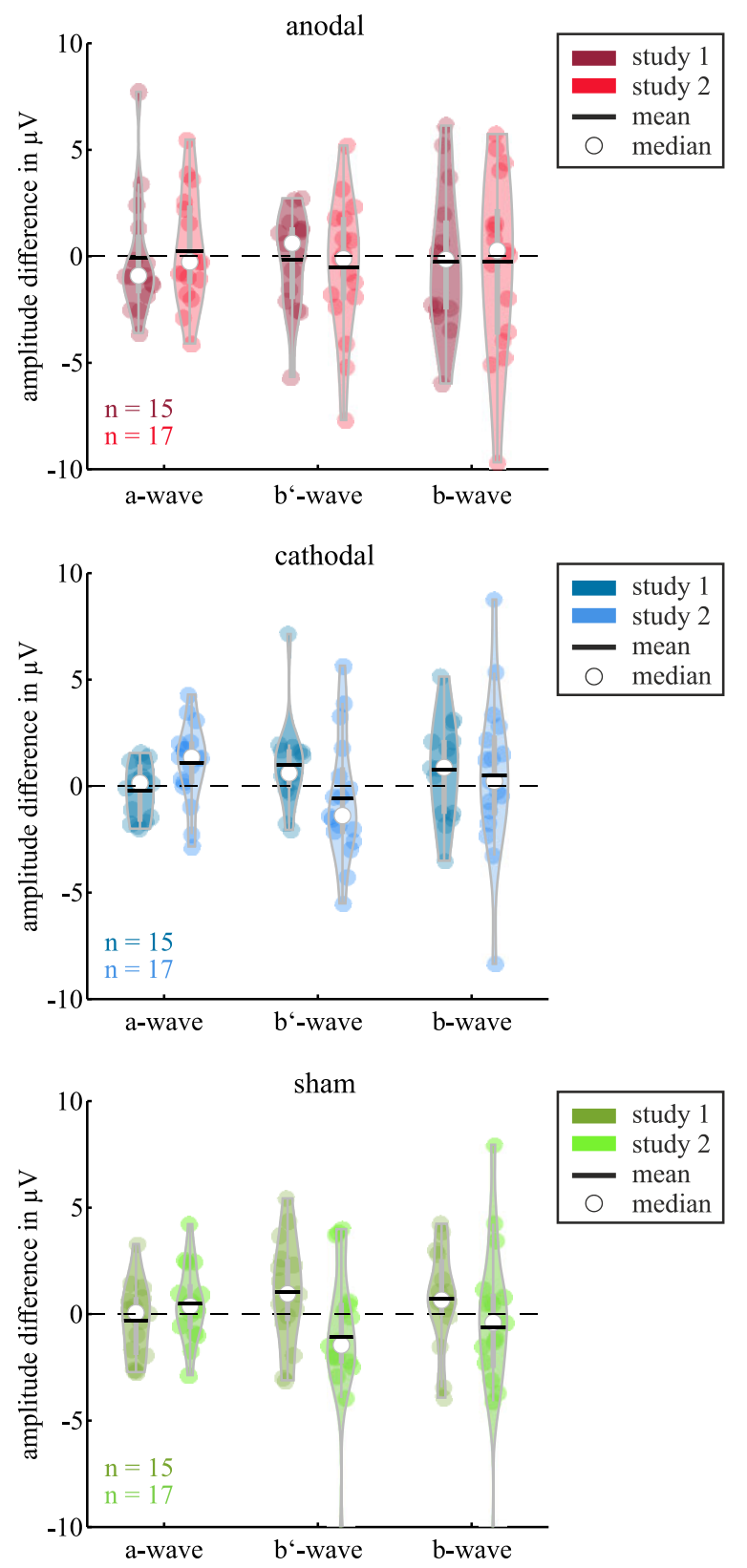

Figure 3: Data distribution for both studies. The violins show the differences between the ERG 1 and ERG 2 measurement for the different current applications, characteristic waves, and studies.
With regard to the stimulation design in both studies, no difference for the ocular CS effect on the ffERG for the comparison between two different CS electrode placements was found. It can be concluded that the retinal cells generating the ffERG are unaffected by the electric fields of an ocular CS tested here. This supports the hypothesis that the known CS effects for the PERG are due to the influence of ganglion cells and not of preganglion cells.

\section{Author Statement}

Research funding: This research was supported by the German Federal Ministry of Education and Research (FKZ: 13GW0331B) as well as from the Studienstiftung des deutschen Volkes. Conflict of interest: Authors state no conflict of interest. Informed consent: Informed consent has been obtained from all individuals included in this study. Ethical approval: The research related to human use complies with all the relevant national regulations, institutional policies and was performed in accordance with the tenets of the Helsinki Declaration, and has been approved by the authors' institutional review board or equivalent committee.

\section{References}

[1] Y. Yokoi and T. Sumiyoshi, "Application of transcranial direct current stimulation to psychiatric disorders : trends and perspectives," Neuropsychiatr. Electrophysiol., vol. 1, no. 10, 2015, doi: 10.1186/s40810-015-0012-x.

J. Liu et al., "Effectiveness of Microcurrent Stimulation in Preserving Retinal Function of Blind Leading Retinal Degeneration and Optic Neuropathy: A Systematic Review," Neuromodulation, vol. 2021, 2021, doi: 10.1111/ner.13414

[3] A. Sehic, S. Guo, K. Cho, R. M. Corraya, D. F. Chen, and T. P. Utheim, "Electrical Stimulation as a Means for Improving Vision," Am. J. Pathol., vol. 186, no. 11, pp. 2783-2797, 2016, doi: 10.1016/j.ajpath.2016.07.017. of an Ocular Direct Electrical Stimulation on PatternReversal Electroretinogram," Front. Neurosci., vol. 14, no. June, pp. 1-9, 2020, doi: 10.3389/fnins.2020.00588.

M.-C. Blum, B. Solf, A. Hunold, and S. Klee, "Effects of Ocular Direct Current Stimulation on Full Field Electroretinogram," Front. Neurosci., vol. 15, no. February, pp. 1-9, 2021, doi: 10.3389/fnins.2021.606557. A. Hunold, S. Freitag, K. Schellhorn, and J. Haueisen, "Simulation of the current density distribution for transcranial electric current stimulation around the eye.," Brain Stimul., vol. 8, no. 2, p. 406, 2015.

A. Jamil et al., "Systematic evaluation of the impact of stimulation intensity on neuroplastic after-effects induced by transcranial direct current stimulation," J. Physiol., vol. 595, no. 4, pp. 1273-1288, 2017, doi: 10.1113/JP272738. 